\title{
CONDITION NUMBER AND ECCENTRICITY OF A CLOSED CONVEX CONE
}

\author{
RENÉ HENRION and ALBERTO SEEGER
}

\begin{abstract}
We discuss some extremality issues concerning the circumradius, the inradius, and the condition number of a closed convex cone in $\mathrm{R}^{n}$. The condition number refers to the ratio between the circumradius and the inradius. We also study the eccentricity of a closed convex cone, which is a coefficient that measures to which extent the circumcenter differs from the incenter.
\end{abstract}

\section{Introduction}

This paper is the last part of a triptych initiated in [7] and continued in [8]. It deals with two concepts related to the geometric nature of a convex cone: the circumradius and the inradius. A few words on notation are in order before we recall these concepts. In the sequel the symbol $\Xi_{n}$ indicates the set of nontrivial closed convex cones in the Euclidean space $\mathrm{R}^{n}$. That a convex cone is nontrivial means that it is different from the singleton $\{0\}$ and different from the whole space $\mathrm{R}^{n}$. For avoiding trivialities we assume that the dimension $n$ is at least three. Some special subsets of $\Xi_{n}$ play a prominent role in the discussion, namely

$$
\begin{aligned}
& \Xi_{n}^{\text {sol }}=\left\{K \in \Xi_{n}: K \text { is solid }\right\}, \\
& \Xi_{n}^{\text {pdd }}=\left\{K \in \Xi_{n}: K \text { is pointed }\right\}, \\
& \Xi_{n}^{\text {reg }}=\left\{K \in \Xi_{n}: K \text { is regular }\right\} .
\end{aligned}
$$

Recall that a closed convex cone is solid if its topological interior is nonempty, and it is pointed if it contains no line. Regularity is understood as the combination of solidity and pointedness.

The inradius of $K \in \Xi_{n}$ is defined as the coefficient

$$
\rho(K)=\sup _{x \in K \cap S_{n}} \operatorname{dist}[x, \partial K],
$$

where $\mathrm{S}_{n}$ is the unit sphere of $\mathrm{R}^{n}, \partial K$ is the boundary of $K$, and $\operatorname{dist}[\cdot, \Omega]$ stands for the distance function to a set $\Omega$. Various interpretations of $\rho(K)$ and 
calculus rules for computing this coefficient have been proposed in [3], [4], [5], [7], [8], [10] and other places. We recall that (1) is a matter of finding the radius and center of a largest ball contained in $K$ :

$$
\begin{aligned}
& \underset{\|x\|=1}{\operatorname{maximize} r} \\
& r \in[0,1] \\
& x+r \mathrm{~B}_{n} \subset K,
\end{aligned}
$$

where $\mathrm{B}_{n}$ denotes the closed unit ball of $\mathrm{R}^{n}$. The coefficient $\rho(K)$ is equal to the optimal value of the maximization problem (2). The function $\rho: \Xi_{n} \rightarrow[0,1]$ is continuous if $\Xi_{n}$ is equipped with the gap metric

$$
\delta\left(K_{1}, K_{2}\right)=\max \left\{\max _{x \in K_{1} \cap \mathrm{s}_{n}} \operatorname{dist}\left[x, K_{2}\right], \max _{x \in K_{2} \cap \mathrm{s}_{n}} \operatorname{dist}\left[x, K_{1}\right]\right\},
$$

or with any other equivalent metric for that matter (cf. [10, Proposition 6.3]). We mention in passing that convergence with respect to the metric $\delta$ is equivalent to convergence in the Painlevé-Kuratowski sense (cf. [14, Proposition 4.4]). This fact will be used on several occasions. If $K \in \Xi_{n}$ is solid, then the solution set

$$
\Pi_{\text {inc }}(K)=\left\{x \in K \cap S_{n}: \operatorname{dist}[x, \partial K]=\rho(K)\right\}
$$

to the variational problem (1) is a singleton. The unique element of this set is denoted by $\pi_{\text {inc }}(K)$ and called the incenter of $K$. Theorem 2.9 of [7] asserts that $\pi_{\text {inc }}: \Xi_{n}^{\text {sol }} \rightarrow \mathrm{R}^{n}$ is a continuous map.

The circumradius of $K \in \Xi_{n}$, denoted by $\mu(K)$, is defined as the optimal value of the minimization problem

$$
\begin{aligned}
& \text { minimize } r \\
& \|x\|=1 \\
& r \in[0,1] \\
& K \subset M(x, r) .
\end{aligned}
$$

Here $M(x, r)$ stands for the closed convex cone generated by the ball $x+r \mathrm{~B}_{n}$, that is,

$$
M(x, r)=\mathrm{cl}\left[\bigcup_{\alpha \geq 0} \alpha\left(x+r \mathrm{~B}_{n}\right)\right] .
$$

The closure operation "cl" is superfluous when $r \neq 1$. If $K \in \Xi_{n}$ is pointed, then

$$
\Pi_{\text {circ }}(K)=\left\{x \in \mathrm{R}^{n}:(x, r) \text { solves (3) }\right\}
$$


is a singleton. The unique element of this set is denoted by $\pi_{\text {circ }}(K)$ and called the circumcenter of $K$. By combining Proposition 4.8 and Theorem 5.2 in [7], one sees that $\mu: \Xi_{n} \rightarrow[0,1]$ and $\pi_{\text {circ }}: \Xi_{n}^{\mathrm{ptd}} \rightarrow \mathrm{R}^{n}$ are continuous functions.

This is all what the reader needs to know for having a good understanding of our work. The organization of the paper is as follows. Section 2 discusses some extremality issues concerning inradii and circumradii. Inspired by the definition of the condition number of a nonsingular matrix, we refer to the ratio

$$
\mathrm{c}(K)=\frac{\mu(K)}{\rho(K)}
$$

as the condition number of a solid cone $K \in \Xi_{n}$. The analysis of this concept is the object of Section 3. A regular cone $K \in \Xi_{n}$ is non-eccentric if $\pi_{\text {inc }}(K)=$ $\pi_{\text {circ }}(K)$, otherwise it is said to be eccentric. The eccentricity is a coefficient that measures the gap between the incenter and the circumcenter:

$$
\mathrm{e}(K)=\left\|\pi_{\text {inc }}(K)-\pi_{\text {circ }}(K)\right\| .
$$

This coefficient is studied in detail in Section 4.

\section{Extremality issues for inradii and circumradii}

\subsection{Preliminary results}

Inradii and circumradii are dual objects. Indeed, for all $K \in \Xi_{n}$ one has

$$
\begin{aligned}
& \mu(K)=\sqrt{1-\left[\rho\left(K^{+}\right)\right]^{2}}, \\
& \rho(K)=\sqrt{1-\left[\mu\left(K^{+}\right)\right]^{2}},
\end{aligned}
$$

where $K^{+}$stands for the dual cone of $K$, i.e.,

$$
K^{+}=\left\{y \in \mathrm{R}^{n}:\langle y, x\rangle \geq 0 \text { for all } x \in K\right\} .
$$

Furthermore,

$$
\begin{aligned}
\Pi_{\mathrm{inc}}(K) & =\Pi_{\mathrm{circ}}\left(K^{+}\right), \\
\Pi_{\mathrm{circ}}(K) & =\Pi_{\mathrm{inc}}\left(K^{+}\right) .
\end{aligned}
$$

These duality relationships have been established in [7, Theorem 5.2]. Of course, the inequality

$$
\rho(K) \leq \mu(K)
$$


holds for any $K \in \Xi_{n}$. The next lemma is easy and consistent with intuition. We mention it only for the sake of subsequent use. Recall that a revolution cone is a set of the form

$$
\Gamma(y, \theta):=\left\{x \in \mathrm{R}^{n}:\langle y, x\rangle \geq\|x\| \cos \theta\right\},
$$

where $y$ is a unit vector of $\mathbf{R}^{n}$. The parameter $\theta \in[0, \pi / 2]$ is referred to as the half-aperture angle of the cone.

Lemma 2.1. For $K \in \Xi_{n}$ the following conditions are equivalent:

(a) $\rho(K)=\mu(K)$.

(b) $K$ is a ball-generated cone.

(c) $K$ is a revolution cone.

Proof. That (b) $\Leftrightarrow$ (c) is mentioned in [6, Section 3.1]. By the way, the equivalence between ball-generated cones and revolution cones holds even in Hilbert spaces. In fact, by combining Lemmas 4.12 and 5.1 in [7] one gets

$$
\begin{aligned}
\Gamma(y, \theta) & =M(y, \sin \theta), \\
M(x, r) & =\Gamma(x, \arcsin r) .
\end{aligned}
$$

For proving (b) $\Rightarrow$ (a) one just needs to observe that

$$
\rho(M(x, r))=\mu(M(x, r))=r
$$

for all $(x, r) \in \mathrm{S}_{n} \times[0,1]$. The proof of (a) $\Rightarrow(\mathrm{b})$ is more subtle. Let $\bar{r}:=$ $\rho(K)=\mu(K)$. We suppose that $\bar{r}>0$, otherwise $K$ is a ray, i.e., a cone generated by a ball of radius 0 . Let $\bar{x}:=\pi_{\text {inc }}(K)$ and $\bar{w} \in \Pi_{\text {circ }}(K)$. In such a case

$$
\bar{x}+\bar{r} \mathrm{~B}_{n} \subset K \subset M(\bar{w}, \bar{r}) .
$$

Since $\rho(M(\bar{w}, \bar{r}))=\bar{r}$, the unit vector $\bar{x}$ must be the incenter of $M(\bar{w}, \bar{r})$. In other words,

$$
\bar{x}=\pi_{\text {inc }}(M(\bar{w}, \bar{r}))=\bar{w} .
$$

Hence, the chain of inclusions in (10) yields $M(\bar{x}, \bar{r})=K=M(\bar{w}, \bar{r})$.

REMARK 2.2. It is well known that the dual of a revolution cone is a revolution cone. Hence, the dual of a ball-generated cone is a ball-generated cone. More precisely,

$$
[M(x, r)]^{+}=M\left(x, \sqrt{1-r^{2}}\right) .
$$

For the reader's convenience we recall below a technical result (cf. [8, Lemma 2.25]) that characterizes the incenter of a solid polyhedral cone. Such 
result will be used on a few occasions. The notation "pos" stands for positive (or convex conic) hull.

LEMMA 2.3. Let $K \in \Xi_{n}$ be a solid polyhedral cone represented by

$$
K=\left\{x \in \mathrm{R}^{n}:\left\langle f_{1}, x\right\rangle \geq 0, \ldots,\left\langle f_{m}, x\right\rangle \geq 0\right\},
$$

where $\left\{f_{i}\right\}_{i=1}^{m}$ is a finite collection of unit vectors in $\mathrm{R}^{n}$. Then $\bar{x}=\pi_{\mathrm{inc}}(K)$ if and only if

$$
\begin{aligned}
& \bar{x} \in K \cap \mathrm{S}_{n}, \\
& \bar{x} \in \operatorname{pos}\left\{f_{i}: i \in I(\bar{x})\right\},
\end{aligned}
$$

where $I(\bar{x})$ is the set of indices $j \in\{1, \ldots, m\}$ such that

$$
\left\langle f_{j}, \bar{x}\right\rangle=\min _{1 \leq i \leq m}\left\langle f_{i}, \bar{x}\right\rangle \text {. }
$$

Furthermore, if $\bar{x}$ is the incenter of $K$, then the minimum in (13) is equal to $\rho(K)$.

As first use of Lemma 2.3 we derive an explicit formula for computing the incenter of a special type of polyhedral cone. Recall that a simplicial cone in $\mathrm{R}^{n}$ is a polyhedral cone generated by a basis of $\mathrm{R}^{n}$. In other words, $K \in \Xi_{n}$ is simplicial if and only if

$$
K=\left\{G \lambda: \lambda \in \mathrm{R}_{+}^{n}\right\}
$$

with $G$ standing for a nonsingular matrix of order $n$. A simplicial cone in $\mathrm{R}^{n}$ is regular and has exactly $n$ facets. General information on simplicial cones and facial analysis can be found in [1].

Theorem 2.4. Suppose that $K \in \Xi_{n}$ is generated by the columns of a nonsingular matrix $G=\left[g_{1}, \ldots, g_{n}\right]$. Let $f_{j}$ denote the $j$-th column of $F=$ $\left(G^{-1}\right)^{T}$. Consider the following statements:

(a) $\left\langle g_{i}, g_{j}\right\rangle \geq 0$ for all $i, j \in\{1, \ldots, n\}$.

(b) $\sum_{j=1}^{n}\left\langle g_{i}, g_{j}\right\rangle\left\|f_{j}\right\| \geq 0$ for all $i \in\{1, \ldots, n\}$.

(c) The incenter of $K$ is equidistant to each facet of $K$.

(d) The incenter of $K$ is equal to the vector

$$
\bar{x}:=\frac{\sum_{i=1}^{n}\left\|f_{i}\right\| g_{i}}{\left\|\sum_{i=1}^{n}\right\| f_{i}\left\|g_{i}\right\|} .
$$

(e) $\rho(K)=\left\|\sum_{i=1}^{n}\right\| f_{i}\left\|g_{i}\right\|^{-1}$.

Then one has (a) $\Rightarrow$ (b) $\Leftrightarrow$ (c) $\Leftrightarrow(\mathrm{d}) \Rightarrow$ (e). 
ProOF. Before starting with the proof itself, observe that

$$
\left\langle f_{j}, g_{i}\right\rangle=\delta_{i j} \quad \text { for all } i, j \in\{1, \ldots, n\},
$$

where $\delta_{i j}$ refers to the Kronecker delta. Hence, the simplicial cone (14) can be rewritten as

$$
K=\left\{x \in \mathrm{R}^{n}:\left\langle\hat{f}_{1}, x\right\rangle \geq 0, \ldots,\left\langle\hat{f}_{n}, x\right\rangle \geq 0\right\}
$$

with $\hat{f}_{j}=\left\|f_{j}\right\|^{-1} f_{j}$. For convenience we split the proof in several parts:

(a) $\Rightarrow$ (b). This implication is obvious.

(b) $\Leftrightarrow$ (d). The vector $\bar{x}$ given by (15) clearly belongs to $K \cap \mathrm{S}_{n}$. Thanks to (16) one has

$$
\left\langle\hat{f}_{j}, \bar{x}\right\rangle=\kappa:=\left\|\sum_{i=1}^{n}\right\| f_{i}\left\|g_{i}\right\|^{-1}
$$

for all $j \in\{1, \ldots, n\}$. Hence,

$$
I(\bar{x}):=\left\{j \in\{1, \ldots, n\}:\left\langle\hat{f}_{j}, \bar{x}\right\rangle=\min _{1 \leq i \leq n}\left\langle\hat{f}_{i}, \bar{x}\right\rangle\right\}
$$

is equal to the whole index set $\{1, \ldots, n\}$. By Lemma 2.3 one has

$$
\begin{aligned}
\bar{x}=\pi_{\text {inc }}(K) & \Leftrightarrow \bar{x} \in \operatorname{pos}\left\{\hat{f}_{1}, \ldots, \hat{f}_{n}\right\} \\
& \Leftrightarrow\left\langle g_{1}, \bar{x}\right\rangle \geq 0, \ldots,\left\langle g_{n}, \bar{x}\right\rangle \geq 0 \\
& \Leftrightarrow \sum_{j=1}^{n}\left\langle g_{i}, g_{j}\right\rangle\left\|f_{j}\right\| \geq 0 \text { for all } i \in\{1, \ldots, n\} .
\end{aligned}
$$

(d) $\Rightarrow$ (e). It follows from (17) and the last part of Lemma 2.3.

(d) $\Rightarrow$ (c). The facets of $K$ are $\mathscr{F}_{j}=K \cap \hat{f}_{j}^{\perp}$ with

$$
\hat{f}_{j}^{\perp}=\left\{x \in \mathrm{R}^{n}:\left\langle\hat{f}_{j}, x\right\rangle=0\right\} .
$$

By assumption the vector $\bar{x}$ given by (15) is the incenter of $K$. The condition (17) shows that $\bar{x}$ is equidistant to each hyperplane $\hat{f}_{j}^{\perp}$, the common distance being $\rho(K)$. Let $\xi_{j}$ denote the orthogonal projection of $\bar{x}$ into $\hat{f}_{j}^{\perp}$. Hence,

$$
\xi_{j}=\bar{x}-\left\langle\hat{f}_{j}, \bar{x}\right\rangle \hat{f}_{j}=\bar{x}-\rho(K) \hat{f}_{j}
$$

and $\left\|\xi_{j}-\bar{x}\right\|=\rho(K)$. It follows that $\xi_{j} \in \mathscr{F}_{j}$ and

$$
\operatorname{dist}\left[\bar{x}, \mathscr{F}_{j}\right]=\operatorname{dist}\left[\bar{x}, \hat{f}_{j}^{\perp}\right]=\rho(K) .
$$

This proves the condition (c). 
(c) $\Rightarrow$ (d). One has $\bar{u}:=\pi_{\mathrm{inc}}(K)=\sum_{i=1}^{n} \lambda_{i} g_{i}$, where each $\lambda_{i}$ is positive. Note that

$$
\rho(K)=\operatorname{dist}[\bar{u}, \partial K]=\min _{1 \leq j \leq n} \frac{\left\langle f_{j}, \bar{u}\right\rangle}{\left\|f_{j}\right\|}=\min _{1 \leq j \leq n} \frac{\lambda_{j}}{\left\|f_{j}\right\|},
$$

where the last equality is due to (16). Hence,

$$
\rho(K)\left\|f_{j}\right\| \leq \lambda_{j}
$$

for all $j \in\{1, \ldots, n\}$. But,

$$
\rho(K)=\operatorname{dist}\left[\bar{u}, \mathscr{F}_{j}\right] \geq \operatorname{dist}\left[\bar{u}, \hat{f}_{j}^{\perp}\right]=\frac{\left\langle f_{j}, \bar{u}\right\rangle}{\left\|f_{j}\right\|}=\frac{\lambda_{j}}{\left\|f_{j}\right\|},
$$

where the first equality is due to the assumption (c). So, (18) is in fact an equality, and therefore

$$
\bar{u}=\rho(K) \sum_{i=1}^{n}\left\|f_{i}\right\| g_{i} .
$$

A due normalization shows that $\bar{u}$ is equal to the vector given by (15).

The condition (a) says that the angle between any pair of generators of $K$ does not exceed $\pi / 2$. This requirement is stronger than (b). To see this, consider the simplicial cone $K$ generated by the columns of

$$
G=\left[\begin{array}{rrr}
1 & -1 & -1 \\
0 & 1 & -2 \\
0 & 0 & 1
\end{array}\right]
$$

The condition (a) is clearly violated. However

$$
F=\left(G^{-1}\right)^{T}=\left[\begin{array}{lll}
1 & 0 & 0 \\
1 & 1 & 0 \\
3 & 2 & 1
\end{array}\right], \quad\left\|f_{1}\right\|=\sqrt{11}, \quad\left\|f_{2}\right\|=\sqrt{5}, \quad\left\|f_{3}\right\|=1,
$$

and a direct computation shows that (b) holds. Thus, one can use (15) for computing the incenter of $K$.

Kelly et al. [15] propose a different concept of "center" for a simplicial cone $K$, namely, any point in the interior of $K$ which is equidistant from each facetal hyperplane. This property is satisfied by the vector $\bar{x}$ given by (15). However, such $\bar{x}$ may not be the incenter of $K$. To see this, consider the simplicial cone $K$ generated by the columns of

$$
G=\left[\begin{array}{rrr}
1 & -1 & -1 \\
0 & 1 & 1 \\
0 & 0 & 1
\end{array}\right]
$$


One has

$$
F=\left(G^{-1}\right)^{T}=\left[\begin{array}{rrr}
1 & 0 & 0 \\
1 & 1 & 0 \\
0 & -1 & 1
\end{array}\right], \quad\left\|f_{1}\right\|=\sqrt{2}, \quad\left\|f_{2}\right\|=\sqrt{2}, \quad\left\|f_{3}\right\|=1,
$$

and the condition (b) is violated. As a consequence, $\pi_{\text {inc }}(K)$ and $\bar{x}$ do not coincide.

Theorem 2.4 can be dualized in order to obtain a formula for computing the circumcenter of a simplicial cone.

Corollary 2.5. Let $K \in \Xi_{n}$ be the simplicial cone generated by the columns of the nonsingular matrix $G=\left[g_{1}, \ldots, g_{n}\right]$. Let $f_{j}$ denote the $j$-th column of $F=\left(G^{-1}\right)^{T}$. Then the following conditions are equivalent:

(a) $\sum_{j=1}^{n}\left\langle f_{i}, f_{j}\right\rangle\left\|g_{j}\right\| \geq 0$ for all $i \in\{1, \ldots, n\}$.

(b) The circumcenter of $K$ is equal to the vector

$$
\bar{y}:=\frac{\sum_{i=1}^{n}\left\|g_{i}\right\| f_{i}}{\left\|\sum_{i=1}^{n}\right\| g_{i}\left\|f_{i}\right\|} .
$$

Proof. We apply Theorem 2.4 to $K^{+}$and use the duality formula (7).

\subsection{Comparing the inradii of $K$ and $K^{+}$}

The theory of solidity and pointedness indices for convex cones has been developed in recent years in [10], [11], [12], [13]. Within the context of such theory one can interpret the inradii of $K$ and $K^{+}$as follows:

$$
\begin{gathered}
\rho(K)=\text { solidity index of } K, \\
\rho\left(K^{+}\right)=\text {pointedness index of } K .
\end{gathered}
$$

The first question addressed in this section is to find a closed convex cone that is as solid and pointed as possible:

$$
\text { maximize } F(K):=\left(\rho(K), \rho\left(K^{+}\right)\right) \quad \text { with respect to } K \in \Xi_{n} \text {. }
$$

Beware that solidity and pointedness are antagonic notions. What we mean by this is that both coefficients $\rho(K)$ and $\rho\left(K^{+}\right)$cannot be large (i.e., near 1) at the same time.

Proposition 2.6 characterizes the Pareto solutions to the above bicriteria optimization problem. By definition, $K \in \Xi_{n}$ is a Pareto solution to (19) if there is no $Q \in \Xi_{n}$ such that

$$
\rho(K) \leq \rho(Q) \text { and } \quad \rho\left(K^{+}\right) \leq \rho\left(Q^{+}\right)
$$

with at least one inequality being strict. 
Proposition 2.6. $K \in \Xi_{n}$ is a Pareto solution to (19) if and only if $K$ is a ball-generated cone.

Proof. As a consequence of (5) and (8) one gets

$$
[\rho(K)]^{2}+\left[\rho\left(K^{+}\right)\right]^{2} \leq 1
$$

for all $K \in \Xi_{n}$. In fact, Corollary 8.4 in [10] asserts something stronger: the image set

$$
F\left(\Xi_{n}\right):=\left\{\left(\rho(K), \rho\left(K^{+}\right)\right): K \in \Xi_{n}\right\}
$$

of the problem (19) is equal to

$$
\Omega=\left\{(r, t) \in \mathrm{R}_{+}^{2}: r^{2}+t^{2} \leq 1\right\} .
$$

Hence, $K \in \Xi_{n}$ is a Pareto solution to (19) if and only if the pair $(\rho(K)$, $\left.\rho\left(K^{+}\right)\right)$lies in the upper right portion of $\Omega$, that is to say,

$$
[\rho(K)]^{2}+\left[\rho\left(K^{+}\right)\right]^{2}=1 .
$$

Lemma 2.1 and the duality formula (5) do the rest of the job.

Besides the inequality (20), is there any other interesting relationship between the inradii of $K$ and $K^{+}$? The next result applies only to simplicial cones. That $K \in \Xi_{n}$ is orthogonal simply means that $K$ a polyhedral cone generated by an orthogonal basis of $\mathrm{R}^{n}$. Every orthogonal cone is simplicial, but not conversely.

TheOREm 2.7. If $K \in \Xi_{n}$ is simplicial, then

$$
\rho(K) \rho\left(K^{+}\right) \leq 1 / n
$$

The above inequality becomes an equality if and only if $K \in \mathbf{\Xi}_{n}$ is orthogonal.

Proof. Let $K \in \Xi_{n}$ be generated by the columns of a nonsingular matrix $G=\left[g_{1}, \ldots, g_{n}\right]$ and let $f_{j}$ denote the $j$-th column of $F=\left(G^{-1}\right)^{T}$. There are vectors $\lambda, \beta$ in $\mathrm{R}_{+}^{n}$ such that

$$
\begin{aligned}
& \bar{u}:=\pi_{\mathrm{inc}}(K)=G \lambda=\sum_{i=1}^{n} \lambda_{i} g_{i}, \\
& \bar{v}:=\pi_{\mathrm{inc}}\left(K^{+}\right)=F \beta=\sum_{i=1}^{n} \beta_{j} f_{j} .
\end{aligned}
$$


It follows that

$$
\begin{gathered}
\rho(K)=\operatorname{dist}[\bar{u}, \partial K]=\min _{1 \leq j \leq n} \frac{\left\langle f_{j}, \bar{u}\right\rangle}{\left\|f_{j}\right\|}=\min _{1 \leq j \leq n} \frac{\lambda_{j}}{\left\|f_{j}\right\|}, \\
\rho\left(K^{+}\right)=\operatorname{dist}\left[\bar{v}, \partial K^{+}\right]=\min _{1 \leq i \leq n} \frac{\left\langle g_{i}, \bar{v}\right\rangle}{\left\|g_{i}\right\|}=\min _{1 \leq i \leq n} \frac{\beta_{i}}{\left\|g_{i}\right\|} .
\end{gathered}
$$

Hence, for all $j \in\{1, \ldots, n\}$ one gets

$$
\begin{array}{r}
\rho(K)\left\|f_{j}\right\| \leq \lambda_{j}, \\
\rho\left(K^{+}\right)\left\|g_{j}\right\| \leq \beta_{j},
\end{array}
$$

and therefore

$$
\rho(K) \rho\left(K^{+}\right)\left(\sum_{j=1}^{n}\left\|f_{j}\right\|\left\|g_{j}\right\|\right) \leq \sum_{j=1}^{n} \lambda_{j} \beta_{j} .
$$

Thanks to (16) and the Cauchy-Schwarz inequality, one has $\left\|f_{j}\right\|\left\|g_{j}\right\| \geq 1$. Hence,

$$
n \leq \sum_{j=1}^{n}\left\|f_{j}\right\|\left\|g_{j}\right\|
$$

For completing the proof of (21) it remains to observe that

$$
\sum_{i=1}^{n} \lambda_{j} \beta_{j}=\left\langle G^{-1} \bar{u}, F^{-1} \bar{v}\right\rangle=\left\langle\bar{u},\left(G^{-1}\right)^{T} F^{-1} \bar{v}\right\rangle=\langle\bar{u}, \bar{v}\rangle
$$

is less than or equal to 1 . If $K \in \Xi_{n}$ is orthogonal, then $\rho(K)=\rho\left(K^{+}\right)=$ $\sqrt{1 / n}$, and $\rho(K) \rho\left(K^{+}\right)=1 / n$. Conversely, let $K=\operatorname{pos}\left\{g_{1}, \ldots, g_{n}\right\}$ be a simplicial cone such that (21) holds as an equality. In such a case

$$
\sum_{j=1}^{n}\left\|f_{j}\right\|\left\|g_{j}\right\|=n
$$

and therefore $\left\|f_{j}\right\|\left\|g_{j}\right\|=1$ for all $j \in\{1, \ldots, n\}$. This and (16) imply that, up to normalization, the sets $\left\{g_{j}\right\}_{j=1}^{n}$ and $\left\{f_{j}\right\}_{j=1}^{n}$ coincide. Hence,

$$
\left\langle\frac{g_{j}}{\left\|g_{j}\right\|}, \frac{g_{i}}{\left\|g_{i}\right\|}\right\rangle=\left\langle\frac{f_{j}}{\left\|f_{j}\right\|}, \frac{g_{i}}{\left\|g_{i}\right\|}\right\rangle=\frac{\left\langle f_{j}, g_{i}\right\rangle}{\left\|f_{j}\right\|\left\|g_{i}\right\|}=0
$$

for all $i \neq j$, that is to say, $K$ is orthogonal. 
Remark 2.8. The product rule (21) does not apply beyond a simplicial context. For instance, the Lorentz (or ice-cream) cone

$$
\Lambda_{n}=\left\{x \in \mathrm{R}^{n}:\left[x_{1}^{2}+\cdots+x_{n-1}^{2}\right]^{1 / 2} \leq x_{n}\right\}
$$

satisfies $\rho\left(\Lambda_{n}\right)=\rho\left(\Lambda_{n}^{+}\right)=\sin (\pi / 4)=\sqrt{1 / 2}$. So, $\rho\left(\Lambda_{n}\right) \rho\left(\Lambda_{n}^{+}\right)=1 / 2$ is greater than $1 / n$.

We now derive a second product rule for inradii. It is less sharp than (21), but applies to arbitrary convex cones. One says that $K \in \Xi_{n}$ is Lorentzian if there exists an orthogonal matrix $U$ of order $n$ such that $K=U\left(\Lambda_{n}\right)$. Equivalently, a Lorentzian cone is a revolution cone with $\pi / 4$ as half-aperture angle.

Proposition 2.9. For any $K \in \Xi_{n}$ one has

$$
\rho(K) \rho\left(K^{+}\right) \leq 1 / 2 .
$$

The above inequality is an equality if and only if $K \in \Xi_{n}$ is Lorentzian.

Proof. The proof of Proposition 2.6 shows that

$$
\rho(K) \rho\left(K^{+}\right) \leq \max _{(r, t) \in \Omega} r t=1 / 2 .
$$

Note that (25) becomes an equality if $K$ is Lorentzian. Indeed, in such a case one has

$$
\rho(K)=\rho\left(K^{+}\right)=\sqrt{1 / 2} .
$$

Conversely, let $K \in \Xi_{n}$ be such that (25) is an equality. Then the pair $\left(\rho(K), \rho\left(K^{+}\right)\right)$solves the maximization problem in (26). This is equivalent to saying that (27) holds. By combining (5) and Lemma 2.1 one deduces that $K$ is generated by a ball. More precisely,

$$
K=M(\bar{x}, \sqrt{1 / 2})
$$

with $\bar{x}=\pi_{\text {inc }}(K)$. But, according to [6], the set (28) is equal to a revolution cone with $\bar{x}$ as revolution axis and $\arcsin (\sqrt{1 / 2})=\pi / 4$ as half-aperture angle. So, up to orthogonal transformation, $K$ is equal to $\Lambda_{n}$. 


\subsection{Inradii and maximal angles}

The next theorem establishes a curious relationship between the circumradius $\mu(K)$ and the maximal angle

$$
\theta_{\max }(K)=\max _{u, v \in K \cap \mathrm{S}_{n}} \arccos \langle u, v\rangle
$$

of $K \in \Xi_{n}$. It also establishes a link between the inradius $\rho(K)$ and the maximal angle of the dual cone $K^{+}$.

TheOREM 2.10. For all $K \in \Xi_{n}$ one has

$$
\begin{aligned}
& {[\mu(K)]^{2} \leq\left(1-\frac{1}{n}\right)\left(1-\cos \left[\theta_{\max }(K)\right]\right),} \\
& {[\rho(K)]^{2} \geq \frac{1}{n}+\left(1-\frac{1}{n}\right) \cos \left[\theta_{\max }\left(K^{+}\right)\right] .}
\end{aligned}
$$

Proof. In view of the duality formula (5), it is enough to prove the relation (30). Suppose that $K \in \Xi_{n}$ is solid, otherwise $\theta_{\max }\left(K^{+}\right)=\pi$ and (30) holds trivially. For convenience we distinguish between two cases.

I. The polyhedral case. Let $K$ be expressible as intersection of finitely many closed half-spaces, that is,

$$
K=\left\{x \in \mathrm{R}^{n}:\left\langle f_{1}, x\right\rangle \geq 0, \ldots,\left\langle f_{m}, x\right\rangle \geq 0\right\} .
$$

Without loss of generality one assumes that

$$
\left\{f_{i}\right\}_{i=1}^{m} \text { is a positively independent collection of unit vectors of } \mathbf{R}^{n} \text {. }
$$

Note that the integer $m$ could be much larger than $n$. According to Lemma 2.3, the incenter $\bar{x}=\pi_{\text {inc }}(K)$ of the solid polyhedral cone (31) satisfies the condition (12) and

$$
\left\langle f_{j}, \bar{x}\right\rangle=\rho(K)
$$

for all $j \in I(\bar{x})$. The conic version of Caratheodory's theorem applied to (12) yields the representation

$$
\bar{x}=\sum_{i \in I} \lambda_{i} f_{i},
$$

where each scalar $\lambda_{i}$ is positive and $I$ is a subset of $I(\bar{x})$ such that $\left\{f_{i}\right\}_{i \in I}$ is linearly independent. The general formulation and proof of the conic version of 
Caratheodory's theorem can be found in numerous references, see for instance Theorem 3.2 in [18, Chapter 1]. From (34) one gets

$$
\left\langle f_{j}, \bar{x}\right\rangle=\sum_{i \in I} \lambda_{i}\left\langle f_{i}, f_{j}\right\rangle
$$

for all $j \in I$, as well as

$$
\langle\bar{x}, \bar{x}\rangle=\sum_{i \in I} \lambda_{i}\left\langle f_{i}, \bar{x}\right\rangle=\rho(K) \sum_{i \in I} \lambda_{i} .
$$

Since $\bar{x}$ has unit length, it follows that

$$
\sum_{i \in I} \lambda_{i}=1 / \rho(K)
$$

Thanks to (33) and (35), for all $j \in I$ one has

$$
\rho(K)=\lambda_{j}+\sum_{i \in I \backslash\{j\}} \lambda_{i}\left\langle f_{i}, f_{j}\right\rangle .
$$

Since the $f_{i}$ are unit vectors in $K^{+}$, one has

$$
\gamma:=\cos \left[\theta_{\max }\left(K^{+}\right)\right] \leq\left\langle f_{i}, f_{j}\right\rangle .
$$

Hence,

$$
\sum_{i \in I \backslash\{j\}} \lambda_{i}\left\langle f_{i}, f_{j}\right\rangle \geq \gamma \sum_{i \in I \backslash\{j\}} \lambda_{i}=\gamma\left(\sum_{i \in I} \lambda_{i}-\lambda_{j}\right)
$$

for all $j \in I$. This, together with (36) and (37), produces the inequality

$$
\rho(K) \geq \lambda_{j}+\gamma\left(\frac{1}{\rho(K)}-\lambda_{j}\right)
$$

for all $j \in I$. By passing to the sum and using (36) again, one obtains

$$
\operatorname{card}(I)\left(\rho(K)-\frac{\gamma}{\rho(K)}\right) \geq \frac{1-\gamma}{\rho(K)} .
$$

But the cardinality of $I$ cannot exceed $n$. Hence, the above line leads to

$$
[\rho(K)]^{2}-\gamma \geq \frac{1-\gamma}{n},
$$

which is just another way of writing (30). 
II. The nonpolyhedral case. Suppose that $K$ is not polyhedral. Theorem 4.4 in [17] asserts that any closed convex cone in an Euclidean space can be written as Painlevé-Kuratowski limit of a sequence of polyhedral cones. As a consequence of this approximation result, there exists a sequence $\left\{K_{\nu}\right\}_{v \in \mathbb{N}}$ of polyhedral cones $K_{v} \in \Xi_{n}$ such that

$$
\lim _{v \rightarrow \infty} \delta\left(K_{v}, K\right)=0 .
$$

But the celebrated Walkup-Wets Isometry Theorem (cf. [19, Theorem 1]) says that the duality operation $Q \mapsto Q^{+}$is an isometry on $\left(\Xi_{n}, \delta\right)$, i.e.,

$$
\delta\left(Q_{1}^{+}, Q_{2}^{+}\right)=\delta\left(Q_{1}, Q_{2}\right) \text { for all } Q_{1}, Q_{2} \in \Sigma_{n} .
$$

Hence, one also has

$$
\lim _{\nu \rightarrow \infty} \delta\left(K_{v}^{+}, K^{+}\right)=0 .
$$

As shown in Part I, for each $v \in \mathrm{N}$ one can write

$$
\left[\rho\left(K_{v}\right)\right]^{2} \geq \frac{1}{n}+\left(1-\frac{1}{n}\right) \cos \left[\theta_{\max }\left(K_{v}^{+}\right)\right] .
$$

Thanks to the continuity of $\rho: \Xi_{n} \rightarrow[0,1]$ and $\theta_{\max }: \Xi_{n}: \rightarrow[0, \pi]$, one has

$$
\lim _{v \rightarrow \infty} \rho\left(K_{v}\right)=\rho(K), \quad \lim _{v \rightarrow \infty} \theta_{\max }\left(K_{v}^{+}\right)=\theta_{\max }\left(K^{+}\right) .
$$

It suffices then to pass to the limit in (38) as $v \rightarrow \infty$.

There are a number of interesting consequences that can be derived from Theorem 2.10. Recall that $K \in \Xi_{n}$ is said to be

$$
\left\{\begin{array}{l}
\text { supradual if } K \supset K^{+}, \\
\text {infradual if } K \subset K^{+}, \\
\text {selfdual if } K=K^{+} .
\end{array}\right.
$$

Clearly, infraduality implies pointedness and supraduality implies solidity. A quantitative version of these statements reads as follows.

Corollary 2.11. One has:

(a) If $K \in \Xi_{n}$ is infradual, then $\mu(K) \leq \sqrt{1-(1 / n)}$.

(b) If $K \in \Xi_{n}$ is supradual, then $\rho(K) \geq \sqrt{1 / n}$.

Proof. $K \in \Xi_{n}$ is infradual if and only if $\theta_{\max }(K) \leq \pi / 2$. Part (a) is then a consequence of (29). Similarly, that $K \in \Xi_{n}$ is supradual is equivalent to saying that $\theta_{\max }\left(K^{+}\right) \leq \pi / 2$. Hence, part (b) is a consequence of (30). 
The lower bound in Corollary 2.11(b) is optimal. Indeed,

$$
\min _{\substack{K \in \Xi_{n} \\ K \text { supradual }}} \rho(K)=\sqrt{1 / n}
$$

with attainment of the minimum if $K \in \Xi_{n}$ is orthogonal. Similarly, the upper bound in Corollary 2.11(a) is optimal because

$$
\max _{\substack{K \in \Xi_{n} \\ K \text { infradual }}} \mu(K)=\sqrt{1-(1 / n)}
$$

with attainment of the maximum at any $K \in \Xi_{n}$ that is orthogonal.

REMARK 2.12. Every orthogonal cone is selfdual. Beware that an arbitrary selfdual cone may not achieve the minimum in (39) or the maximum in (40). To see this, consider the cone $K$ generated by the vectors

$$
\left[\begin{array}{l}
1 \\
1 \\
1
\end{array}\right],\left[\begin{array}{l}
0 \\
1 \\
1
\end{array}\right], \quad\left[\begin{array}{r}
-1 \\
0 \\
1
\end{array}\right],\left[\begin{array}{r}
0 \\
-1 \\
1
\end{array}\right],\left[\begin{array}{r}
1 \\
-1 \\
1
\end{array}\right] .
$$

This cone is proposed by Barker and Foran [2] as example of self-dual polyhedral cone that is not simplicial. On the other hand, it is shown in [8, Example 2.3] that $\rho(K)>\sqrt{1 / 3}$, which means that $K$ does not achieve the minimum in (39).

For the sake of completeness we mention another result in the same vein as Corollary 2.11.

Corollary 2.13. Let $K \in \Xi_{n}$.

(a) If $K$ is infradual, then $\rho(K) \leq \sqrt{1 / 2}$.

(b) If $K$ is simplicial and infradual, then $\rho(K) \leq \sqrt{1 / n}$.

(c) If $K$ is supradual, then $\mu(K) \geq \sqrt{1 / 2}$.

(d) If $K$ is simplicial and supradual, then $\mu(K) \geq \sqrt{1-(1 / n) \text {. }}$

Proof. Part (a) is a consequence of (5) and (8). Part (b) follows from Theorem 2.7. Parts (c) and (d) are obtained by applying (a) and (b) to the dual cone $K^{+}$.

\section{Condition number of a convex cone}

As mentioned before, the condition number of a solid cone $K \in \Xi_{n}$ is defined as the ratio $\mathrm{c}(K)=\mu(K) / \rho(K)$. By mimicking the parlance of numerical linear algebra, one says that $K$ is well-conditioned if $\mathrm{c}(K)$ is near to 1 and ill-conditioned if $\mathrm{c}(K)$ is much larger than 1 . 
Example 3.1. Consider the elliptic cone

$$
\mathscr{E}_{A}=\left\{(z, t) \in \mathrm{R}^{n-1} \times \mathrm{R}: \sqrt{\langle z, A z\rangle} \leq t\right\}
$$

associated to a positive definite symmetric matrix $A$ of order $n-1$. Proposition 6.4 in [10] asserts that

$$
\begin{aligned}
& \rho\left(\mathscr{E}_{A}\right)=\left[1+\lambda_{\max }(A)\right]^{-1 / 2}, \\
& \mu\left(\mathscr{E}_{A}\right)=\left[1+\lambda_{\min }(A)\right]^{-1 / 2},
\end{aligned}
$$

where $\lambda_{\max }(A)$ and $\lambda_{\min }(A)$ denote, respectively, the largest and smallest eigenvalue of $A$. Hence,

$$
\mathrm{c}\left(\mathscr{E}_{A}\right)=\sqrt{\frac{1+\lambda_{\max }(A)}{1+\lambda_{\min }(A)}} .
$$

Note that $\mathrm{c}\left(\mathscr{E}_{A}\right)=1$ if and only if $A$ is a positive multiple of the identity matrix.

EXAMPLE 3.2. As a generalization of the above example, consider the epigraph

$$
\text { epi } \phi=\left\{(z, t) \in \mathrm{R}^{n-1} \times \mathrm{R}: \phi(z) \leq t\right\}
$$

of a norm $\phi$ on $\mathrm{R}^{n-1}$. Such set is clearly a regular cone in $\mathrm{R}^{n}$. Lemmas 3.1 and 4.1 in [16] assert, respectively, that

$$
\begin{aligned}
& \rho(\text { epi } \phi)=\left[1+\beta_{\phi}^{2}\right]^{-1 / 2}, \\
& \mu(\operatorname{epi} \phi)=\alpha_{\phi}\left[1+\alpha_{\phi}^{2}\right]^{-1 / 2},
\end{aligned}
$$

where

$$
\alpha_{\phi}:=\min _{\|z\|=1} \phi(z) \quad \text { and } \quad \beta_{\phi}:=\max _{\|z\|=1} \phi(z)
$$

Hence,

$$
\mathrm{c}(\operatorname{epi} \phi)=\sqrt{\frac{1+\beta_{\phi}^{2}}{1+\alpha_{\phi}^{2}}} .
$$

Note that $\mathrm{c}(\operatorname{epi} \phi)=1$ if and only if $\phi$ is constant on the unit sphere $S_{n-1}$.

What does it mean actually that $c(K)$ is near to 1 ? The next theorem provides an answer to this question. We establish first a topological lemma concerning the collection

$$
\Xi_{n}^{\text {ball }}=\left\{M(x, r):(x, r) \in \mathrm{S}_{n} \times[0,1]\right\}
$$

of ball-generated cones in $\mathrm{R}^{n}$. 
LEMMA 3.3. $\Xi_{n}^{\text {ball }}$ is a closed set in the metric space $\left(\Xi_{n}, \delta\right)$.

Proof. The lemma is surely known, so we give only a sketch of the proof. Let $\left\{K_{v}\right\}_{v \in \mathrm{N}}$ be a sequence in $\Xi_{n}^{\text {ball }}$ such that $\lim _{v \rightarrow \infty} \delta\left(K_{v}, K\right)=0$. For each $v \in \mathrm{N}$ one has $K_{v}=M\left(x_{v}, r_{v}\right)$ with $\left(x_{v}, r_{v}\right) \in \mathrm{S}_{n} \times[0,1]$. By taking a subsequence if necessary, one may suppose that

$$
\lim _{v \rightarrow \infty}\left(x_{v}, r_{v}\right)=(\hat{x}, \hat{r}) \in \mathrm{S}_{n} \times[0,1]
$$

A routinary work shows that

$$
\limsup _{v \rightarrow \infty} M\left(x_{v}, r_{v}\right) \subset M(\hat{x}, \hat{r}) \subset \liminf _{v \rightarrow \infty} M\left(x_{v}, r_{v}\right),
$$

where the upper and lower limits are understood in the Painlevé-Kuratowski sense. One proves in this way that $K=M(\hat{x}, \hat{r})$. Hence, $K \in \Xi_{n}^{\text {ball }}$.

In view of Lemma 2.1, a solid cone $K \in \Xi_{n}$ satisfies c $(K)=1$ if and only if $K \in \Xi_{n}^{\text {ball }}$. An asymptotic version of this statement is formulated in the next theorem. The notation

$$
\operatorname{dist}\left[K, \Xi_{n}^{\text {ball }}\right]:=\min _{M \in \Xi_{n}^{\text {ball }}} \delta(K, M)
$$

indicates the distance from $K \in \Xi_{n}$ to the closed set $\Xi_{n}^{\text {ball }}$. Since the metric space $\left(\Xi_{n}, \delta\right)$ is compact (cf. [9, Proposition 2.1]), the subset $\Xi_{n}^{\text {ball }}$ is compact as well. This explains why the minimum in (41) is attained.

THEOREM 3.4. Let $\left\{K_{v}\right\}_{v \in \mathrm{N}}$ be a sequence in $\Xi_{n}$ satisfying the Uniform Solidity Condition

$$
\inf _{v \in \mathrm{N}} \rho\left(K_{v}\right)>0
$$

Then

$$
\lim _{\nu \rightarrow \infty} \mathrm{c}\left(K_{v}\right)=1 \Longleftrightarrow \lim _{\nu \rightarrow \infty} \operatorname{dist}\left[K_{v}, \Xi_{n}^{\text {ball }}\right]=0
$$

Proof. Let us start with the "if" part. Suppose that

$$
d_{v}:=\operatorname{dist}\left[K_{v}, \Xi_{n}^{\text {ball }}\right]
$$

goes to 0 as $v \rightarrow \infty$. For each $v \in \mathrm{N}$, pick $\left(x_{v}, r_{v}\right) \in \mathrm{S}_{n} \times[0,1]$ such that $M_{v}=M\left(x_{v}, r_{v}\right)$ achieves the distance from $K_{v}$ to $\Xi_{n}^{\text {ball }}$, that is,

$$
\delta\left(K_{v}, M_{v}\right)=d_{\nu}
$$


As shown in [10, Proposition 6.3], the inradius $\rho$ is a nonexpansive function on $\left(\Xi_{n}, \delta\right)$. Hence,

$$
\left|\rho\left(K_{v}\right)-\rho\left(M_{\nu}\right)\right| \leq \delta\left(K_{v}, M_{v}\right) .
$$

By combining (9), (43), and (44), one gets

$$
\rho\left(K_{v}\right)=\rho\left(M_{\nu}\right)+\varepsilon_{v}=r_{v}+\varepsilon_{v}
$$

with $\left\{\varepsilon_{v}\right\}_{v \in \mathrm{N}}$ converging to 0 . We now examine the term $\mu\left(K_{v}\right)$. The nonexpansiveness of $\rho$ and the Walkup-Wets Isometry Theorem yield

$$
\left|\rho\left(K_{v}^{+}\right)-\rho\left(M_{v}^{+}\right)\right| \leq \delta\left(K_{v}^{+}, M_{v}^{+}\right)=\delta\left(K_{v}, M_{v}\right) .
$$

But

$$
M_{v}^{+}=\left[M\left(x_{v}, r_{v}\right)\right]^{+}=M\left(x_{v}, \sqrt{1-r_{v}^{2}}\right) .
$$

One gets in this way

$$
\rho\left(K_{v}^{+}\right)=\rho\left(M_{v}^{+}\right)+\gamma_{v}=\sqrt{1-r_{v}^{2}}+\gamma_{v}
$$

with $\left\{\gamma_{v}\right\}_{v \in N}$ converging to 0 . Thanks to the duality relation (5), one arrives at

$$
\mu\left(K_{v}\right)=\left(1-\left[\rho\left(K_{v}^{+}\right)\right]^{2}\right)^{1 / 2}=\left(1-\left[\sqrt{1-r_{v}^{2}}+\gamma_{v}\right]^{2}\right)^{1 / 2} .
$$

We must show that

$$
\mathrm{c}\left(K_{v}\right)=\frac{\left(1-\left[\sqrt{1-r_{v}^{2}}+\gamma_{v}\right]^{2}\right)^{1 / 2}}{r_{v}+\varepsilon_{v}}
$$

goes to 1 as $v \rightarrow \infty$. In view of (45), the Uniform Solidity Condition (42) implies that the sequence $\left\{r_{v}\right\}_{v \in \mathrm{N}}$ remains away from 0 . Hence, the numerator and denominator of the quotient (46) are asymptotically equal. More precisely, both behave as $r_{v}$. We now prove the "only if" part. Suppose that $\lim _{v \rightarrow \infty} \mathrm{c}\left(K_{v}\right)=1$. We claim that the upper limit

$$
\Delta=\limsup _{\nu \rightarrow \infty} \operatorname{dist}\left[K_{v}, \Xi_{n}^{\text {ball }}\right]
$$

is equal to zero. Let $\varphi: \mathrm{N} \rightarrow \mathrm{N}$ be an increasing function such that

$$
\lim _{\nu \rightarrow \infty} \operatorname{dist}\left[K_{\varphi(\nu)}, \Xi_{n}^{\text {ball }}\right]=\Delta .
$$


Since $\left\{K_{\varphi(v)}\right\}_{v \in \mathrm{N}}$ lies in the compact metric space $\left(\Xi_{n}, \delta\right)$, there exists yet another increasing function $\psi: \mathrm{N} \rightarrow \mathrm{N}$ and an element $\widetilde{K} \in \Xi_{n}$ such that

$$
\lim _{\nu \rightarrow \infty} \delta\left(Q_{\nu}, \tilde{K}\right)=0
$$

with $Q_{\nu}=K_{\varphi(\psi(v))}$. Let $r_{\nu}=\rho\left(Q_{v}\right)$ and $s_{\nu}=\mu\left(Q_{v}\right)$. Then

$$
M\left(x_{v}, r_{v}\right) \subset Q_{v} \subset M\left(w_{v}, s_{v}\right)
$$

with $x_{v}=\pi_{\text {inc }}\left(Q_{v}\right)$ and $w_{v} \in \Pi_{\text {circ }}\left(Q_{v}\right)$. Since $\rho, \mu$ are continuous functions on $\Xi_{n}$, one gets

$$
\begin{aligned}
\bar{r} & :=\lim _{\nu \rightarrow \infty} r_{\nu}=\rho(\tilde{K}) \\
\bar{s} & :=\lim _{\nu \rightarrow \infty} s_{\nu}=\mu(\tilde{K}) .
\end{aligned}
$$

The Uniform Solidity Condition (42) implies that $\bar{r}>0$. Hence, $\widetilde{K}$ is solid and

$$
1=\lim _{\nu \rightarrow \infty} \mathrm{c}\left(K_{v}\right)=\lim _{\nu \rightarrow \infty} \mathrm{c}\left(Q_{\nu}\right)=\lim _{\nu \rightarrow \infty} s_{\nu} / r_{v}=\bar{s} / \bar{r},
$$

that is to say, $\bar{s}=\bar{r}$. Given that $\left\{x_{v}\right\}_{v \in \mathrm{N}}$ and $\left\{w_{\nu}\right\}_{v \in \mathrm{N}}$ are in the compact set $\mathrm{S}_{n}$, there is an increasing function $\phi: \mathrm{N} \rightarrow \mathrm{N}$ such that both limits

$$
\bar{x}=\lim _{v \rightarrow \infty} x_{\phi(v)}, \quad \bar{w}=\lim _{v \rightarrow \infty} w_{\phi(v)}
$$

exist. By continuity arguments one obtains $\bar{x}=\pi_{\text {inc }}(\widetilde{K})$ and $\bar{w} \in \Pi_{\text {circ }}(\widetilde{K})$. Now, passing to Painlevé-Kuratowski limits in the sandwich

$$
M\left(x_{\phi(v)}, r_{\phi(v)}\right) \subset Q_{\phi(v)} \subset M\left(w_{\phi(v)}, s_{\phi(v)}\right),
$$

and keeping in mind that $\bar{s}=\bar{r}$, one arrives at

$$
\bar{x}+\bar{r} \mathrm{~B}_{n} \subset M(\bar{x}, \bar{r}) \subset \widetilde{K} \subset M(\bar{w}, \bar{r}) .
$$

We are in the same situation as in (10), so one deduces that $\widetilde{K}=M(\bar{w}, \bar{r})$ is a ball-generated cone. Hence,

$$
\begin{aligned}
\Delta & =\lim _{\nu \rightarrow \infty} \operatorname{dist}\left[K_{\varphi(v)}, \Xi_{n}^{\text {ball }}\right]=\lim _{\nu \rightarrow \infty} \operatorname{dist}\left[Q_{\nu}, \Xi_{n}^{\text {ball }}\right] \\
& =\lim _{\nu \rightarrow \infty} \operatorname{dist}\left[\widetilde{K}, \Xi_{n}^{\text {ball }}\right]=0 .
\end{aligned}
$$

This proves our claim and completes the proof of the theorem. 


\section{Eccentricity of a regular cone}

The eccentricity of a regular cone $K \in \Xi_{n}$ is defined by the expression (4), that is to say, it is the gap between the incenter and the circumcenter of $K$. By squaring both sides of (4) and keeping in mind that $\pi_{\text {inc }}(K)$ and $\pi_{\text {circ }}(K)$ are unit vectors, one gets

$$
[\mathrm{e}(K)]^{2}=\left\|\pi_{\text {inc }}(K)-\pi_{\text {circ }}(K)\right\|^{2}=2-2 a(K),
$$

where $\mathrm{a}(K):=\left\langle\pi_{\text {inc }}(K), \pi_{\text {circ }}(K)\right\rangle$. In other words, the eccentricity

$$
\mathrm{e}(K)=\sqrt{2(1-\mathrm{a}(K))}
$$

of a regular cone $K$ has to do also with the angle formed by $\pi_{\text {inc }}(K)$ and $\pi_{\text {circ }}(K)$.

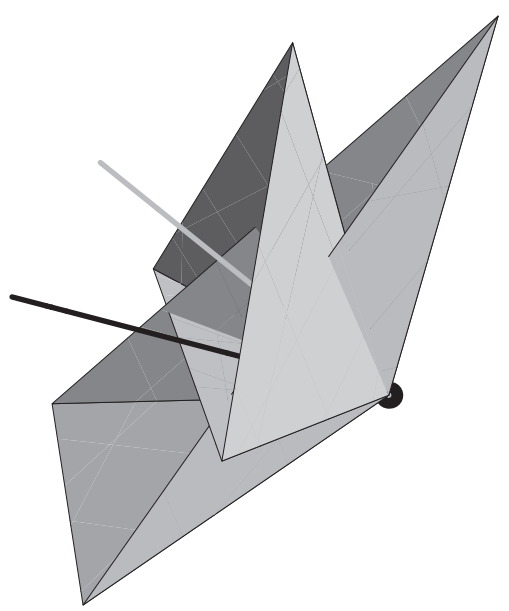

FIgURE 1. Mutually dual simplicial cones in $\mathrm{R}^{3}$. One ray corresponds to the incenter of $K$, the other ray corresponds to the circumcenter of $K$ (i.e., the incenter of $\left.K^{+}\right)$. In this example the angle between $\pi_{\text {inc }}(K)$ and $\pi_{\text {circ }}(K)$ is almost $\pi / 4$, suggesting a rather high degree of eccentricity.

Proposition 4.1. The function e : $\Xi_{n}^{\mathrm{reg}} \rightarrow \mathrm{R}$ is continuous and satisfies the following properties:

(a) $\mathrm{e}\left(K^{+}\right)=\mathrm{e}(K)$ for all $K \in \Xi_{n}^{\mathrm{reg}}$.

(b) $0 \leq \mathrm{e}(K)<\sqrt{2}$ for all $K \in \Xi_{n}^{\mathrm{reg}}$.

(c) $\left\{\mathrm{e}(K): K \in \Xi_{n}^{\mathrm{reg}}\right\}$ is an interval.

PROOF. Both functions $\pi_{\text {inc }}$ and $\pi_{\text {circ }}$ are continuous on $\Xi_{n}^{\text {reg }}$. Part (a) is a consequence of (6) and (7). The strict inequality in (b) follows from (47) and 
the fact that a $(K)>0$ for all $K \in \Xi_{n}^{\text {reg }}$. Recall that $\pi_{\text {inc }}(K)$ belongs to the interior of $K$ and $\pi_{\text {circ }}(K)$ belongs to the interior of $K^{+}$. The set in (c) is an interval because $\Xi_{n}^{\text {reg }}$ is arc-connected (cf. [9, Proposition 7.3]).

The next theorem provides an upper bound for the eccentricity in terms of the coefficient

$$
\Phi(K)=\frac{\rho(K)+\rho\left(K^{+}\right)}{1+\rho(K) \rho\left(K^{+}\right)} .
$$

Such an expression is well defined for all $K \in \Xi_{n}$ and satisfies

$$
0 \leq \max \left\{\rho(K), \rho\left(K^{+}\right)\right\} \leq \Phi(K) \leq 1
$$

These inequalities are all strict when $K$ is regular.

THEOREM 4.2. For all $K \in \Xi_{n}^{\text {reg }}$ one has

$$
\mathrm{e}(K)<\sqrt{2(1-\Phi(K))}
$$

Furthermore, there exists sequence $\left\{K_{v}\right\}_{v \in \mathrm{N}}$ in $\Xi_{n}^{\text {reg }}$ such that

$$
\sqrt{2\left(1-\Phi\left(K^{v}\right)\right)}-\mathrm{e}\left(K^{v}\right) \rightarrow 0 \text { as } v \rightarrow \infty
$$

Proof. We claim that the inner product of $x=\pi_{\text {inc }}(K)$ and $y=\pi_{\text {circ }}(K)$ is greater than $\Phi(K)$. We suppose that $x \neq y$, otherwise we are done. If one sets $r=\rho(K)$ and $s=\mu(K)$, then one can write

$$
x+r \mathrm{~B}_{n} \subset K \subset M(y, s) .
$$

Hence, for all $u \in \mathrm{B}_{n}$ one has $x+r u \in M(y, s)$ or, equivalently,

$$
t\|x+r u\| \leq\langle y, x+r u\rangle
$$

with $t=\sqrt{1-s^{2}}=\rho\left(K^{+}\right)$. We exploit the relation (50) for the particular choice $u=-y$. Since $x$ and $y$ are not collinear, one has

$$
\|x-r y\|>1-r\langle x, y\rangle,
$$

and therefore

$$
t(1-r\langle x, y\rangle)<\langle x, y\rangle-r .
$$

After simplification one obtains

$$
\langle x, y\rangle>\frac{r+t}{1+r t} .
$$


This confirms our claim and completes the proof of (49). For proving the last part of the theorem we consider a revolution cone

$$
K_{v}=\left\{w \in \mathrm{R}^{n}:(1 / v)\|w\| \leq\langle z, w\rangle\right\}
$$

whose revolution axis is a given vector $z \in \mathrm{S}_{n}$. For each $v \geq 1$ one has

$$
\pi_{\text {inc }}\left(K_{v}\right)=\pi_{\text {circ }}\left(K_{v}\right)=z,
$$

and therefore $\mathrm{e}\left(K_{v}\right)=0$. On the other hand, $\rho\left(K_{v}\right)=\left[1-(1 / v)^{2}\right]^{1 / 2}$ and $\rho\left(K_{v}^{+}\right)=1 / v$, so $\Phi\left(K_{v}\right) \rightarrow 1$ as $v$ goes to infinity.

Keeping in mind (48) one gets in particular

$$
\begin{aligned}
& \mathrm{e}(K)<\sqrt{2(1-\rho(K))}, \\
& \mathrm{e}(K)<\sqrt{2\left(1-\rho\left(K^{+}\right)\right)}
\end{aligned}
$$

for all $K \in \Xi_{n}^{\text {reg }}$. However, these upper bounds are less sharp than (49).

COROLLARY 4.3. If $K \in \Xi_{n}^{\text {reg }}$ is either infradual or supradual, then

$$
\mathrm{e}(K)<\sqrt{2\left(1-n^{-1 / 2}\right)}
$$

Proof. If $K \in \Xi_{n}^{\text {reg }}$ is supradual, then $\rho(K) \geq \sqrt{1 / n}$ by Corollary 2.11 . The relation (53) is then a consequence of (51). If $K \in \Xi_{n}^{\text {reg }}$ is infradual, then $\rho\left(K^{+}\right) \geq \sqrt{1 / n}$ and (53) is a consequence of (52).

The following theorem proposes an alternative to the upper bound (49), but it concerns only the class of simplicial cones. Note that the new bound (54) is exact for orthogonal cones, whereas (49) is not.

Theorem 4.4. If $K \in \mathbf{\Xi}_{n}$ is simplicial, then

$$
\mathrm{e}(K) \leq \sqrt{2\left(1-n \rho(K) \rho\left(K^{+}\right)\right)} .
$$

Proof. Consider again the proof of Theorem 2.7. By combining (22), (23), and (24), one gets

$$
n \rho(K) \rho\left(K^{+}\right) \leq\left\langle\pi_{\text {inc }}(K), \pi_{\text {inc }}\left(K^{+}\right)\right\rangle .
$$

But

$$
\left\langle\pi_{\text {inc }}(K), \pi_{\text {inc }}\left(K^{+}\right)\right\rangle=\left\langle\pi_{\text {inc }}(K), \pi_{\text {circ }}(K)\right\rangle=1-(1 / 2)[\mathrm{e}(K)]^{2} .
$$

This yields the announced relation (54). 
Though we know that the eccentricity of a regular cone is smaller that $\sqrt{2}$, it remains an open question to compute

$$
E_{n}=\sup \left\{\mathrm{e}(K): K \in \Xi_{n}^{\mathrm{reg}}\right\} .
$$

It is not clear whether this supremum depends on $n$ and which one is its exact value. For the sake of illustration we give below an example showing that

$$
\sqrt{2-\sqrt{2}} \leq E_{3} \leq \sqrt{2}
$$

ExAmple 4.5. For each $v \geq 2$, let $K_{v}$ be the simplicial cone generated by the columns of

$$
G_{v}=\left[\begin{array}{ccc}
0 & v^{-1} & v^{-1} \\
0 & \sqrt{1-v^{-2}} & 0 \\
1 & 0 & \sqrt{1-v^{-2}}
\end{array}\right]
$$

A long and tedious computation shows that $\lim _{v \rightarrow \infty} \mathrm{e}\left(K_{v}\right)=\sqrt{2-\sqrt{2}}$, which explains the lower bound in (55).

\section{REFERENCES}

1. Barker, G. P., Theory of cones, Linear Algebra Appl. 39 (1981), 263-291.

2. Barker, G. P., and Foran, J., Self-dual cones in Euclidean spaces, Linear Algebra Appl. 13 (1976), 147-155.

3. Epelman, M., and Freund, R. M., A new condition measure, preconditioners, and relations between different measures of conditioning for conic linear systems, SIAM J. Optim. 12 (2002), 627-655.

4. Freund, R. M., On the primal-dual geometry of level sets in linear and conic optimization, SIAM J. Optim. 13 (2003), 1004-1013.

5. Freund, R. M., and Vera, J. R., Condition-based complexity of convex optimization in conic linear form via the ellipsoid algorithm, SIAM J. Optim. 10 (1999), 155-176.

6. Goffin, J. L., The relaxation method for solving systems of linear inequalities, Math. Oper. Res. 5 (1980), 388-414.

7. Henrion, R., and Seeger, A., On properties of different notions of centers for convex cones, Set-Valued Var. Anal. 18 (2010), 205-231.

8. Henrion, R., and Seeger, A., Inradius and circumradius of various convex cones arising in applications, Set-Valued Var. Anal. 18 (2010), 483-511.

9. Iusem, A., and Seeger, A., Pointedness, connectedness, and convergence results in the space of closed convex cones, J. Convex Anal. 11 (2004), 267-284.

10. Iusem, A., and Seeger, A., Axiomatization of the index of pointedness for closed convex cones, Comput. Applied Math. 24 (2005), 245-283.

11. Iusem, A., and Seeger, A., Measuring the degree of pointedness of a closed convex cone: a metric approach, Math. Nachr. 279 (2006), 599-618.

12. Iusem, A., and Seeger, A., Computing the radius of pointedness of a convex cone, Math. Program. 111 (2008), 217-241. 
13. Iusem, A., and Seeger, A., Normality and modulability indices I: Convex cones in normed spaces, J. Math. Anal. Appl. 338 (2008), 392-406.

14. Iusem, A., and Seeger, A., Distances between closed convex cones: old and new results, J. Convex Anal. 17 (2010), 1033-1055.

15. Kelly, L. M., Murty, K. G., and Watson, L. T., CP-rays in simplicial cones, Math. Program. 48 (1990), 387-414.

16. Seeger, A., Epigraphical cones I, J. Convex Anal. 18 (2011), 1179-1196.

17. Seeger, A., Epigraphical cones II, J. Convex Anal. 19 (2012), online Jan. 2011.

18. Matasov, A. I., Estimators for Uncertain Dynamic Systems, Math. Appl. 458, Kluwer, Dordrecht 1998.

19. Walkup, D. W., and Wets, R.J.-B., Continuity of some convex-cone-valued mappings, Proc. Amer. Math. Soc. 18 (1967), 229-235.

WEIERSTRASS INSTITUTE FOR APPLIED ANALYSIS AND STOCHASTICS MOHRENSTRASSE 39

10117 BERLIN

GERMANY

E-mail: henrion@wias-berlin.de
UNIVERSITY OF AVIGNON

DEPARTMENT OF MATHEMATICS

33 RUE LOUIS PASTEUR

84000 AVIGNON

FRANCE

E-mail: alberto.seeger@univ-avignon.fr 